\title{
Early Results of Laparoscopic Sleeve Gastrectomy for Treatment of Morbid Obesity : Experience of Surgery Unit 8b- Ain Shams University, Egypt
}

\author{
Ahmed Hussein Abdelhafez, MD; Mohammed Shaaban Khalifa, MD; \\ Mohammed Mostafa Marzouk, MD.
}

\author{
Department of General Surgery, Faculty of Medicine, Ain Shams University, \\ Cairo, Egypt.
}

Background: Sleeve gastrectomy (SG) is a relatively new bariatric procedure with a number of advantages compared with Roux-en-Y gastric bypass. However, SG also has a number of disadvantages and associated risks. We sought to examine perioperative complications and early outcomes of laparoscopic $S G(L S G)$.

Patients and methods: Since July 2013, LSG has been performed at surgery unit8Bdepartment of surgery, Ain Shams University, Cairo, Egypt, and we reviewed the cases of all patients. We conducted a retrospective review in September 2015.

Results: Forty patients had LSG, and none was lost to follow-up. Indications for LSG over other bariatric procedures were patient preference $80 \%(n=32)$, severe obesity with a body mass index (BMI) greater than $60 \mathrm{~kg} / \mathrm{m}^{2}(n=5)$ and severe upper abdominal adhesions $(n=3) .28$ patients out of forty were women, while the other 12 were men; and the average age was 38 (standard deviation [SD] 10) years. Preoperatively, the average BMI was 50.3 $\left(S D\right.$ 7.7) $\mathrm{kg} / \mathrm{m}^{2}$. Preoperative obesity-related comorbidity rates were $25 \%(n=10)$ for type 2 diabetes mellitus (T2DM), 50\% $(n=20)$ for hypertension, $20 \%(n=8)$ for dyslipidemia, 10\% $(n=4)$ for obstructive sleep apnea (OSA), 30\% $(n=12)$ for knee and/or hip pain and 37.5\% $(n=15)$ for depression and/or anxiety. The mean duration of surgery was 78 (SD 21) minutes. There were 2 major perioperative complications: 1 staple line leak and 1 gastric pouch (sleeve) stricture. The median stay in hospital was 3 days. Postoperative upper gastrointestinal imaging studies were conducted in all patients; 1 was positive for staple line leak. Histopathology on the excised gastric segments revealed chronic helicobacter pylori gastritis in 4 patients. The mean postoperative follow-up interval was 10 months. Weight loss averaged 28.4 (SD 9.3) kg. Overall weight loss was 3.7 (SD 1.8) $\mathrm{kg} /$ month. Resolution occurred in 60\% of patients with T2DM, 40\% with hypertension, 25\% with dyslipidemia, 75\% with OSA, 25\% with joint pain and $80 \%$ with depression/anxiety. Overall satisfaction was rated as excellent by $60 \%$ of patients $(n=24)$, good by $25 \%(n=10)$ and poor by $15 \%$ of patients $(n=6)$.

Conclusion: Preliminary analysis of our experience with LSG indicates that this is an effective and safe procedure for the treatment of obesity.

Key words: Morbid obesity, laparoscopic sleeve gastrectomy, co-morbidity

\section{Introduction:}

Sleeve gastrectomy (SG) is a relatively new bariatric procedure involving resection of most of the stomach along the greater curvature to leave only a narrow tube ("sleeve") between the gastro-esophageal junction and pylorus.1,2 The remainder of the gastrointestinal tract is not altered. The procedure is typically performed laparoscopically. Weight loss following SG is thought to be due to decreased food intake secondary to reduced stomach volume and 
distensability $^{3}$ and possibly modulation of gastrointestinal hormones. ${ }^{4}$ Recent systematic reviews of bariatric procedures found that SG is comparable to Roux-en-Y gastric bypass (RYGP) with respect to weight loss and improvement in the components of the metabolic syndrome.5,6 Compared with RYGP, SG has several advantages. The relative simplicity of SG results in a shorter duration of surgery and fewer complications. The pylorus is preserved in SG, so patients are less likely to experience dumping syndrome. In SG, the small bowel and mesentery are not altered; as such, there are fewer nutritional deficiencies, there is no added risk of internal hernia, and the entire upper gastrointestinal tract remains accessible for endoscopy. ${ }^{7}$

A further advantage of SG is that there is no permanent large foreign body installed as in adjustable gastric banding (AGB), another popular bariatric procedure. In addition to the usual risks associated with surgery in general and in obese patients in particular, there are disadvantages and risks associated with SG compared with other bariatric techniques. Unlike AGB, SG is irreversible, and there is a risk of gastric stenosis requiring treatment with dilators. The sleeve may become permanently dilated with overeating. Since the lumen cannot be easily adjusted as in AGB, a second malabsorptive procedure such as RYGP may have to be performed to promote further weight loss. There is a risk of leak in the long gastric staple line in SG, which can be fatal if not detected and repaired early. The SG procedure was first described in 1937 and was performed laparoscopically starting about a decade ago as the first of a 2-stage procedure in high-risk obese patients. 8 The SG procedure helped these patients lose some weight and reduce their operative risk before undergoing the more complicated biliopancreatic diversion with duodenal switch. More recently, SG has been increasingly performed on lower-risk obese patients and was recently recognized by the American Society for Metabolic and Bariatric Surgery as a primary (single-stage) bariatric procedure.

\section{Patients and methods:}

Starting in July 2013, selected patients were offered LSG as one of the surgical options for treatment of their obesity. Selection was based on patient preference or contraindications for other bariatric procedures. Counselling and monitoring of diet, exercise and behavior modification was conducted throughout the pre- and postoperative periods. Patients were also encouraged to attend weight loss support groups both before and after surgery, as there is emerging evidence that such support can increase weight loss in the long-term.9,10

Operative technique: All patients underwent upper gastrointestinal endoscopy before LSG to rule out anatomic anomalies, gastric mucosal pathology and Helicobacter pylori infection, as recommended by the Standards of Practice Committee of the American Society for Gastrointestinal Endoscopy. ${ }^{11}$

Preoperatively, patients received prophylactic bowel cleansing, antibiotics and subcutaneous heparin and were placed in pneumatic stockings. The surgical technique involved the placement of 5 trocars as follows: Left upper quadrant (5- to 10-mm $30^{\circ}$ laparoscope), right upper quadrant (5to $12-\mathrm{mm}$ Versa port [Covidien]), and right lateral subcostal (5-mm liver retractor), left subcostal (5 mm) and the epigastrium ( $5 \mathrm{~mm})$. A carbon dioxide pneumoperitoneum was created in the left upper quadrant. The liver was retracted superiorly. Beginning 6-7 cm proximal to the pylorus, the greater curvature of the stomach was freed from the gastrocolic ligament and the short gastric vessels divided with the LigaSure (Covidien) system up to the angle of His Figure (1-A). To create the sleeve, the Endo GIA (Covidien) stapler was fired along a line parallel to the lesser curvature beginning 6-7 $\mathrm{cm}$ proximal to the pylorus up to the cardia such that the sleeve was about 36-F Figure (1-B). In some cases a bougie (34- to $36-\mathrm{F}$ ) placed in the stomach against the lesser curvature was used to guide the stapling. Bleeding from stapling line was observed in 3 cases and dealt with properly with clips applied directly over the bleeding points. The excised portion of the stomach 
was removed from the abdominal cavity by minimally enlarging the incision where the Versa port cannula was placed Figure (1-C), and was sent for pathologic analysis. Patients early in the series received postoperative upper gastrointestinal imaging with watersoluble contrast to check for staple line leaks Figure (2). All patients received follow-up care and had at least 1 follow up visit with their surgeons. The interval for surgeon followup was variable to accommodate the travel schedule of the many patients who resided far away from our bariatric surgical center. A retrospective chart review was conducted in April 2015 on all patients who underwent the LSG. Data collected and analyzed from the charts included demographics, preoperative and current anthropometrics, preoperative and current obesity-related comorbidities and their severities, operative data and a survey of overall patient satisfaction with the procedure. Weight loss was assessed with percent excess weight loss (\%EWL) for comparison to existing literature as well as percent excess body mass index (BMI) loss (\%EBL), which has been proposed as a superior metric for comparison of bariatric procedure outcomes. (9) But is not yet widely used. The \%EWL and \%EBL were calculated using the definitions provided by Deitel and colleagues. ${ }^{12}$

Assessment of changes in co-morbidity status of type 2 diabetes mellitus (T2DM), hypertension and dyslipidemia was defined as follows. Resolution was defined as normalization of the metrics defining each pathology without use of medication. Improvement was defined as either improved control of said metrics while on the same dose of medication or continued adequate control of metrics while on a reduced amount of medication. Resolution of obstructive sleep apnea (OSA) was determined by follow-up assessment by pulmonologists or internists. ${ }^{13}$

Changes in the status of joint pain and mood disorders were assessed subjectively by eliciting the patient's symptoms and perceptions of whether the problem was resolved or improved ${ }^{14}$ Also weight loss is charted and precisely assessed and recorded at 1 month, 3 months, 6 months, 9 months, 12 months (1 year), 18 months and two years postoperatively Figure (3).

\section{Results:}

Forty patients underwent LSG performed between July 2013 and December 2014, and none was lost to follow-up. 28 patients out of 40 were women and the other 12 were men; mean age was 38 (SD 10, range 23-57) years. The mean preoperative BMI was 50.3 (SD 7.7, range 39-63) kg/m². Most patients $(80 \%, \mathrm{n}=32)$ chose LSG over other bariatric procedures, whereas the remaining patients had LSG because of contraindications, including severe obesity with BMI over $60 \mathrm{~kg} / \mathrm{m}^{2}(\mathrm{n}=5)$ and severe upper abdominal adhesions $(n=3)$, to other procedures. The preoperative obesity-related comorbidity rates were $25 \%(\mathrm{n}=10)$ for T2DM, $50 \%$ $(\mathrm{n}=20)$ for hypertension, 20\% $(\mathrm{n}=8)$ for dyslipidemia, $10 \%(\mathrm{n}=4)$ for OSA, 30\% $(\mathrm{n}=12)$ for $\mathrm{knee}$ and/or hip pain and $37.5 \%$ $(n=15)$ for depression and/or anxiety Table (1). The mean duration of surgery was 78 (SD 21, range 45-125) minutes. The median number of stapler firings was 6 (range 5-9). The staples and LigaSure system formed the bulk of the surgical materials cost. (6 firings, plus 1 LigaSure). All resected gastric specimens were sent for pathologic examination. Despite negative preoperative endoscopy and biopsy, histopathology of all resected portions of the stomach revealed 4 cases of chronic H. Pylori gastritis. The median length of stay in hospital was 3 days (range 1-12 d). Only one patient stayed 1 week due to staple line leak and discharged after completing resolution. There was no perioperative or postoperative mortality.

Patients had postoperative upper gastrointestinal imaging studies with watersoluble contrast when it was clinically indicated to check for staple line leak. One case had a high gastric staple line leak detected by radiological and clinical data and underwent reoperation within 48 hours of initial surgery to over sew the leak via laparotomy (open surgery) and endoscopic stent placement was done post laparotomy. The patient was treated 
with TPN and antibiotics. Three patients had staple line hemorrhage intraoperatively and secured via clips applied directly over bleeding points with prompt hemostasis. One patient had severe gastric stricture (stenosis) with repeated vomiting, upper GI contrast and endoscopy reflected tight gastric Pouch. Patients had failed attempt of endoscopic dilatation and explored via midline laparotomy and had open gastrojejunostomy due to laparoscopic technical difficulties.The mean follow-up time with the bariatric surgeon was 10 (range 2-24) months. . Pre-and postoperative obesityrelated comorbidity rates and changes among patients who underwent laparoscopic sleeve gastrectomy are summarized in Table (1). Overall mean weight loss was 28.4 (SD 9.3, range $13.6-34.4) \mathrm{kg}$ and monthly mean weight loss was3.7 (SD 1.8, range 0.38.6) $\mathrm{kg} /$ month. Overall mean \%EWL was $35.4 \%$ (SD $13.8 \%$, range $6.5 \%-68.6 \%$ ) and monthly mean $\%$ EWL was $4.1 \%$ (SD $2.4 \%$, range $0.5 \%-11.4 \%$ ) per month. Overall mean $\%$ EBL was $39.4 \%$ (SD15.8\%, range $8.7 \%-95.2 \%$ ) and monthly mean $\%$ EBL was $3.8 \%$ (SD $2.6 \%$, range $0.5 \%-13.6 \%$ ) per month. There was postoperative resolution or improvement in several components of the metabolic syndrome: Diabetes resolved in $60 \%(n=6)$ and improved in $40 \%(n=4)$, hypertension resolved in $40 \%(\mathrm{n}=8)$ and improved in $60 \%(\mathrm{n}=12)$, and dyslipidemia resolved in 25\% $(\mathrm{n}=2)$ and improved in 50\% $(\mathrm{n}=4)$. Obstructive sleep apnea resolved in $75 \%(\mathrm{n}=3)$ and improved in $25 \%(\mathrm{n}=1)$, joint pain resolution occurred in $25 \%(n=3)$ and improved in $75 \%(n=9)$ with resolution in depression/anxiety patients or improvement $80 \%(\mathrm{n}=12)$. With regard to overall patient satisfaction with LSG, 60\% $(n=24)$ rated their experience as excellent, 25\% $(n=10)$ rated it as good, and $15 \%(\mathrm{n}=6)$ rated it as poor.

\section{Discussion:}

We reviewed the cases of all 40 patients who underwent LSG since we began offering this option for bariatric surgery in July 2013. The indication for LSG was patient preference in most cases, which has become the most frequent indication reported in the worldwide literature. ${ }^{6}$ Overall patient satisfaction with the procedure has been high, even among our 2 patients who had major perioperative complications. Both of these patients made a full recovery and achieved substantial weight loss. Besides the general complications associated with surgery, the most frequent perioperative complications associated with LSG include staple line leaks $(2.5 \%)(\mathrm{n}=1)$ and gastric stenosis $(2.5 \%)(\mathrm{n}=1) .(2,6)$ There is no strong evidence for preoperative upper GI endoscopy and its use is variable. The use of a bougie to guide creation of the sleeve is helpful in avoiding stenosis. However, insertion of a bougie may be difficult, and a staple line disruption may occur if care is not taken. We reinforce the staple line by oversewing where indicated. ${ }^{15}$ Postoperative upper gastro-intestinal imaging with watersoluble contrast can be used to check for staple line leaks, although its sensitivity and specificity have been questioned. ${ }^{2}$

We performed postoperative upper gastrointestinal imaging in our early patients, but now order such imaging only when clinically indicated. ${ }^{16}$ There was substantial variability in weight loss in our longer followup groups. This may be an effect of our small sample size, and we hope that more data will help to determine what factors distinguish those who achieve substantial weight loss versus those who do not. In addition to weight loss, reduction in obesity-related comorbidities is another important outcome in bariatric surgery. A recent review of SG reported a high rate of resolution of several components of the metabolic syndrome at postoperative intervals ranging from 12 to 60 months. ${ }^{16}$

The mean and standard deviations of mean resolution rates were $55.7 \%$ (SD $37.3 \%$; 754 patients, 10 studies) for T2DM, 49.6\% (SD 24.6\%; 733 patients, 9 studies) for hypertension and $43.0 \%$ (SD 24.0\%; 513 patients, 6 studies) for dyslipidemia. ${ }^{17}$ There was resolution of T2DM in $60 \%$ of our patients, and many of these patients experienced resolution less than 6 months 


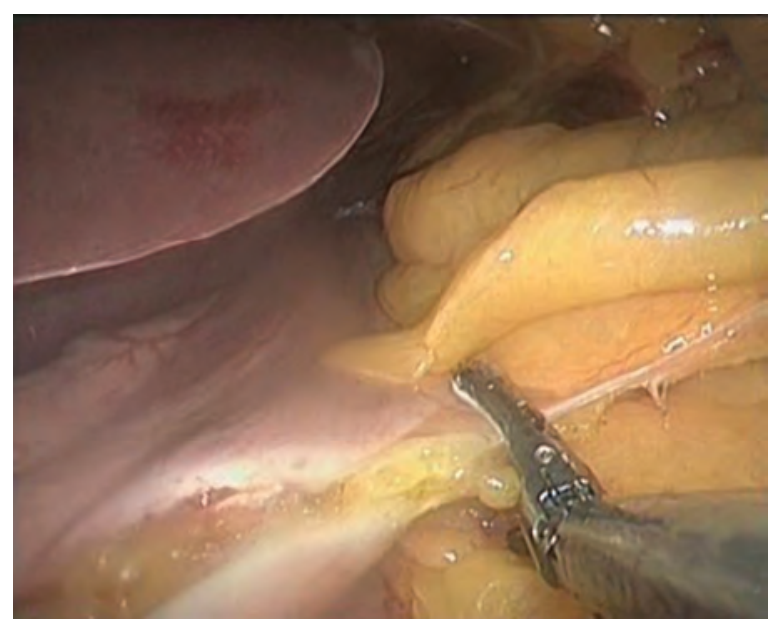

Figure (1-A): Division of gastrocolic ligament and short gastric vessels.

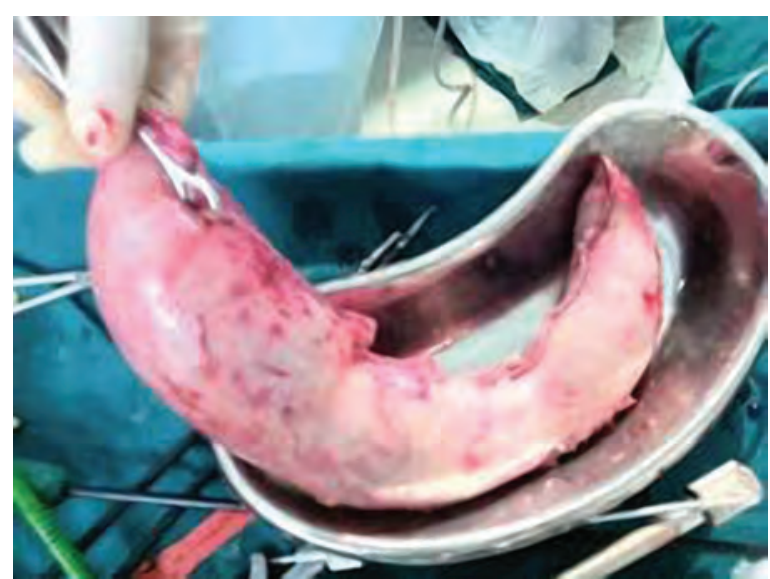

(Figure 1-C): Excised gastric portion.

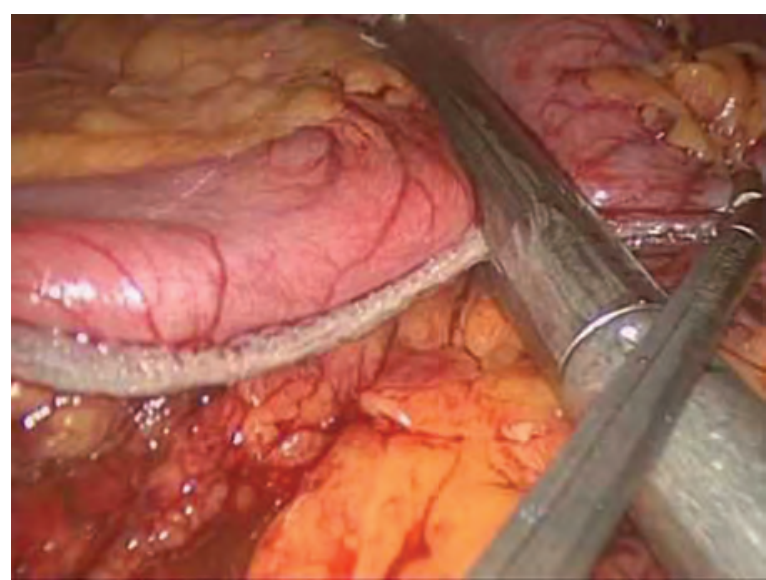

Figure (1-B): Creation of sleeve along a line parallel to the lesser curvature.

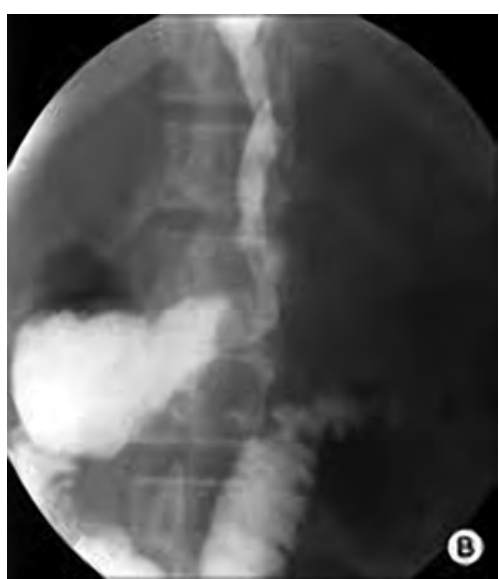

Figure (2): Upper GI contrast(gastrograffin meal)done postoperatively.

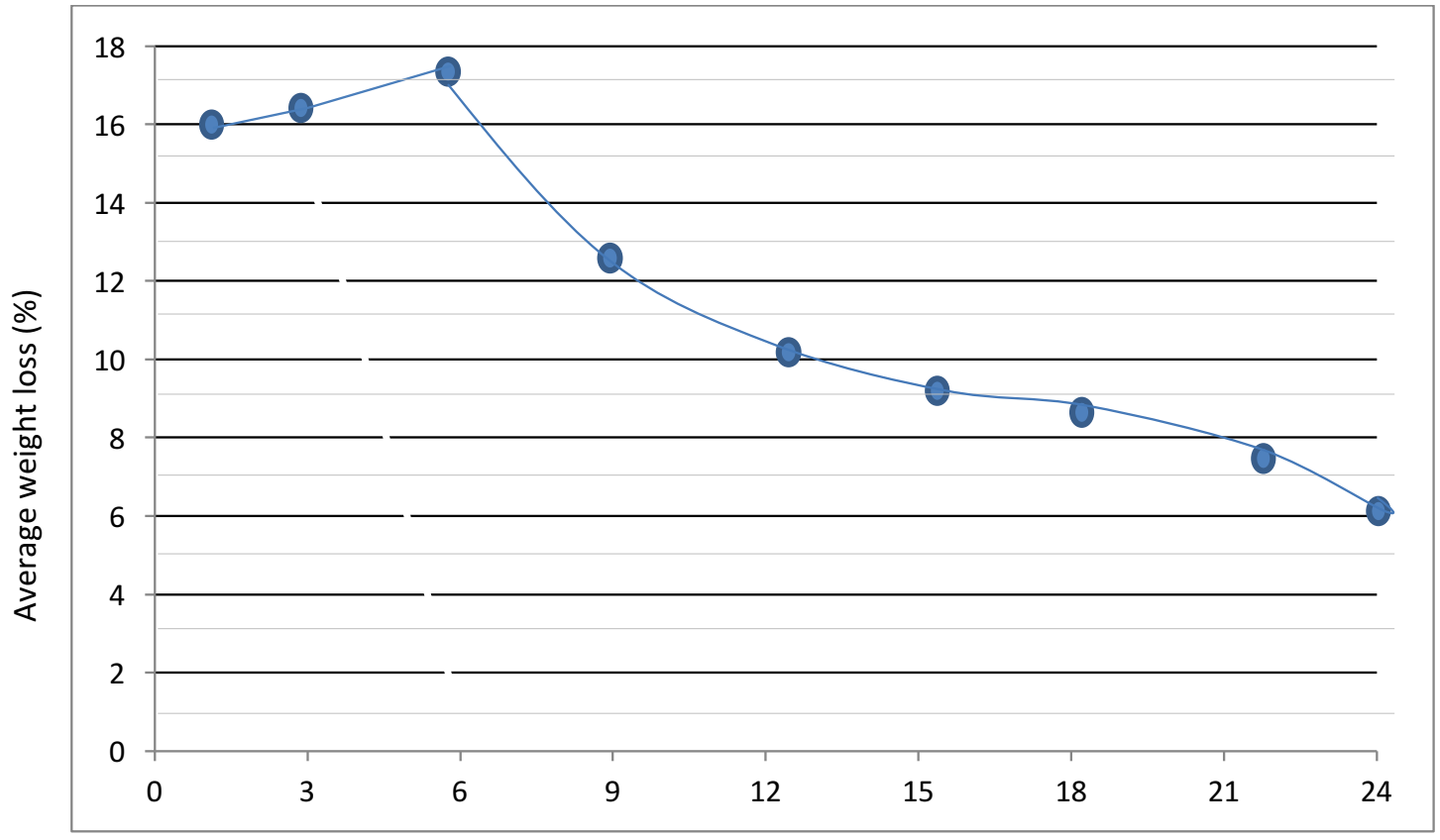

Postoperative period per month interval

Graphic (1): Relationship between percentage average weight loss, and postoperative period per month interval. 
Table 1. Pre-and postoperative obesity-related comorbidity rates and changes among patients who underwent laparoscopic sleeve gastrectomy

\begin{tabular}{|c|c|c|c|c|c|}
\hline \multirow{2}{*}{ Comorbidity } & Preoperative no. (\%) & \multicolumn{3}{|c|}{ Postoperative; no. } \\
\cline { 2 - 6 } & & \multicolumn{3}{|c|}{ Resolved Improved Unchanged } & \\
\hline Type 2 diabetes mellitus & 10 & $(25)$ & $6(60 \%)$ & $4(40 \%)$ & 0 \\
\hline Dyslipidemia & 8 & $(20)$ & $2(25 \%)$ & $4(50 \%)$ & 2 \\
\hline Hypertension & 20 & $(50)$ & $8(40 \%)$ & $12(60 \%)$ & 0 \\
\hline Obstructive sleep apnea & 4 & $(10)$ & $3(75 \%)$ & $1(25 \%)$ & 0 \\
\hline Knee/hip pain & 12 & $(30)$ & $3(25 \%)$ & $9(75 \%)$ & 0 \\
\hline Depression/anxiety & 15 & $(37.5 \%)$ & $(80 \%)$ & 3 \\
\hline
\end{tabular}

after surgery. Resolution of T2DM occurred among patients with \%EWL between $20 \%$ and $67 \%$. The changes in T2DM status observed in our patient population demonstrate the independence of T2DM resolution from weight loss as reported in the literature. ${ }^{15}$ Hypertension resolved in $40 \%$ of our patients and this only occurred in patients with \%EWL over $35 \%$. The $75 \%$ resolution rate of OSA in our patient population was consistent with results reported in the literature. ${ }^{17}$

\section{Conclusion:}

Early results of a series of 40 patients who underwent LSG demonstrated low perioperative complication rates, encouraging results with respect to weight loss and obesity -related comorbidity reduction and high patient satisfaction. Our early results and data from SG literature support our expectation for continuing improvement in health and quality of life for our obese patients. Larger studies and longer follow-up intervals are needed to validate short-term results.

\section{Reference:}

1- Kueper MA, Kramer KM, Kirschniak A: Laparoscopic sleeve gastrectomy: Standardized technique of a potential standalone bariatric procedure in morbidly obese patients. World J Surg 2008; 32: 1462-1465.

2- Gagner M, Deitel M, Kalberer TL: The second international consensus summit for sleeve gastrectomy, March 19-21, 2009. Surg Obes Relat Dis 2009; 5: 476-485.

3- Yehoshua RT, Eidelman LA, Stein M, et al: Laparoscopic sleeve gastrectomy — volume and pressure assessment. Obes Surg 2008;
18: 1083-1088.

4- Wang Y, Liu J: Plasma ghrelin modulation in gastric band operation and sleeve gastrectomy. Obes Surg 2009; 19: 357-362.

5- Colquitt JL, Picot J, Loveman E, et al: Surgery for obesity. Cochrane Database Syst Rev 2008; (4): CD003641.

6- Brethauer SA, Hammell JP, Schauer PR: Systematic review of sleeve gastrectomy as staging and primary bariatric procedure. Surg Obes Relat Dis 2009; 5: 469-475.

7- Marceau P, Biron S, Bourque R-A: Biliopancreatic diversion with a new type of gastrectomy. Obes Surg 1993; 3: 29-35.

8- Hess DS, Hess DW: Biliopancreatic diversion with a duodenal switch. Obes Surg 1998; 8: 267-282.

9- Song Z, Reinhardt K, Buzdon M: Association between support group attendance and weight loss after Roux-en-Y gastric bypass. Surg Obes Relat Dis 2008; 4: 100-103.

10- Elakkary E, Elhorr A, Aziz F: Do support groups play a role in weight loss after laparoscopic gastric banding? Obes Surg 2006; 16: 331-334.

11- ASGE Standards of Practice Committee, Anderson MA, Gan SI, Fanelli RD: Role of endoscopy in the bariatric surgery patient. Gastrointest Endosc 2008; 68: 1-10.

12- Deitel M, Gawdat K, Melissas J: Reporting weight loss 2007 [editorial]. Obes Surg 2007; 17: 565-568.

13- Chen B, Kiriakopoulos A, Tsakayannis D: Reinforcement does not necessarily reduce the rate of staple line leaks after sleeve gastrectomy. A review of the literature and clinical experiences. Obes Surg 2009; 19: 166-172.

14- Dapri G, Cadiere GB, Himpens J: Reinforcing the staple line during laparoscopic sleeve 
gastrectomy: Prospective randomized clinical study comparing three different techniques. Obes Surg 2010; 20: 462-467.

15- Vidal J, Ibarzabal A, Romero F: Type 2 diabetes mellitus and the metabolic syndrome following sleeve gastrectomy in severely obese subjects. Obes Surg 2008; 18 : 1077-1082.
16- Birmingham CL, Muller JL, Palepu A: The cost of obesity in Canada. CMAJ 1999; 160: 483-488.

17- Harris SB, Leiter LA, Yale J-F: Out-ofpocket costs of managing hyperglycemia and hypoglycemia in patients with type 1 diabetes and insulin-treated type 2 diabetes. Can J Diabetes 2007; 31: 25-33. 